Western University

Scholarship@Western

Medical Biophysics Publications

Medical Biophysics Department

$3-12-2015$

Evidence of ventricular contamination of the optical signal in preterm neonates with post hemorrhagic ventricle dilation

J Kishimoto

Western University

M Diop

Western University

S de Ribaupierre

Western University

DSC Lee

KSt. Lawrence

Western University

Follow this and additional works at: https://ir.lib.uwo.ca/biophysicspub

Part of the Medical Biophysics Commons

Citation of this paper:

Kishimoto, J; Diop, M; de Ribaupierre, S; Lee, DSC; and St. Lawrence, K, "Evidence of ventricular contamination of the optical signal in preterm neonates with post hemorrhagic ventricle dilation" (2015). Medical Biophysics Publications. 61.

https://ir.lib.uwo.ca/biophysicspub/61 


\title{
Evidence of ventricular contamination of the optical signal in preterm neonates with post hemorrhagic ventricle dilation
}

\author{
J Kishimoto $^{1,2}$, M Diop ${ }^{1,2}$, P McLachlan ${ }^{1,2}$, S de Ribaupierre ${ }^{1,3}$, DSC Lee ${ }^{4}$, K St Lawrence ${ }^{1,2}$ \\ ${ }^{1}$ Lawson Health Research Institute, London, Ontario, Canada \\ ${ }^{2}$ Department of Medical Biophysics, Western University, London, Ontario, Canada \\ ${ }^{3}$ Department of Clinical Neurological Sciences, Western University, London, Ontario, Canada \\ ${ }^{4}$ Division of Neonatal-Perinatal Medicine, Department of Paediatrics, Western University, London, \\ Ontario, Canada
}

Keywords: near infrared spectroscopy, neonate, intraventricular hemorrhage, ventricle dilation, blood break down products, preterm, hydrocephalus

\begin{abstract}
S
Dilation of the cerebral ventricles is a common condition in preterm neonates with intraventricular hemorrhage (IVH). This post hemorrhagic ventricle dilation (PHVD) can lead to lifelong neurological impairment through ischemic injury due to increased intracranial pressure (ICP). Interventions, such as ventricular tapping to remove cerebrospinal fluid (CSF), are used to prevent injury, but determining the optimal time for treatment is difficult as clinical signs of increased ICP lack sensitivity. There is a growing interest in using near-infrared spectroscopy (NIRS) because of its ability to monitor cerebral oxygen saturation $\left(\mathrm{S}_{\mathrm{t}} \mathrm{O}_{2}\right)$ at the bedside. However, the accuracy of NIRS may be affected by signal contamination from enlarged ventricles, especially if there are blood breakdown products (bbp) in CSF following IVH. To investigate this, serial NIR spectra from the head and from CSF samples were acquired over a month from seven IVH patients undergoing treatment for PHVD. Over time, the visual appearance of the CSF samples progressed from dark brown ("tea color") to clear yellow, reflecting the reduction in bbp concentration as confirmed by the stronger absorption around 760 $\mathrm{nm}$ at the earlier time points. All CSF samples contained strong absorption at $960 \mathrm{~nm}$ due to water. More importantly the same trend in these absorption features was observed in the in vivo spectra, and Monte Carlo simulations confirmed the potential for signal contamination from enlarged ventricles. These findings highlight the challenges of accurately measuring $\mathrm{S}_{\mathrm{t}} \mathrm{O}_{2}$ in this patient population and the necessity of using a hyperspectral NIRS system to resolve the additional chromophores.
\end{abstract}

\section{INTRODUCTION}

Preterm neonates born very early at low birth weight $(<1500 \mathrm{~g})$ commonly have brain bleeds inside the ventricles, called intraventricular hemorrhage or IVH, and it is estimated to effect 10-30\% of these patients.[1] IVH is thought to be caused by a combination of poor autoregulation and a fragile germinal matrix (the highly vascularized region from which brain cells migrate during development).[2] Bleeding can cause dilation of the cerebral ventricles, called post hemorrhagic ventricle dilation (PHVD), through blood clots which block the 'flow' of CSF from the ventricles to the arachnoid space where it is reabsorbed into the blood stream. While mild IVH is generally associated with mild PHVD that eventually will resolve without intervention, severe IVH can result in persistent, progressive enlargement of the ventricles, called hydrocephalus, which is associated with a greater risk of brain injury and many morbidities later in life. $[3,4]$

Since preterm neonates are often incubator bound, they are not good candidates to be moved to an imaging facility for more traditional 3D images such as CT or MRI. Patients at risk of neurological impairment, such as those with PHVD, are currently monitored using serial 2D ultrasound (US) to detect ventricular enlargement. [5] Additionally, patients are monitored for increased intracranial pressure (ICP), which in neonates with non-sutured skull plates presents as tense/bulging fontanelles ('soft spots') and abnormal expansion of the head (macroencephaly), which is measured through serial head circumference measurements.[6] However, these indicators do not have the specificity to guide surgical interventions aimed at preventing brain injury, as these symptoms often present after injury has already occurred.

Cerebral monitoring using near infrared spectroscopy (NIRS) is a promising technology for PVHD monitoring considering it can be performed at the bedside and should be capable of detecting changes in cerebral oxygenation and

Optical Tomography and Spectroscopy of Tissue XI, edited by Bruce J. Tromberg, Arjun G. Yodh,

Eva Marie Sevick-Muraca, Robert R. Alfano, Proc. of SPIE Vol. 9319, 931908

(c) 2015 SPIE · CCC code: 1605-7422/15/\$18 - doi: 10.1117/12.2077232 
blood flow caused by elevated ICP. Previous work in IVH patients has been primarily targeted at monitoring oxygenation saturation very early in life.[7-9] There has been additional work looking at patients with PHVD who required intervention and acute changes in hemodynamic indicators showed improvement immediately after the intervention.[10] To our knowledge no NIRS study on PHVD patients has been done longitudinally over the entire course of a NICU stay.

In this paper we will describe potential sources of signal contamination seen in NIRS measurements of PHVD patients which could affect longitudinal monitoring. Specifically, the effect of 'ventricular contamination' due to decreased cortical thickness associated with PHVD was examined. We obtained both cerebral spectra from preterm patients with 'normal' brains as well as the abnormal spectra from patients with IVH using a hyperspectral continuous wave (CW) NIRS system. Ultrasound images and NIRS spectra were acquired early in life (within the first month), and any time an interventional ventricle tap (VT) was required clinically. Cerebral spinal fluid (CSF) spectra were obtained after VT to determine the amount of blood breakdown product (bbp) within the ventricles. This in turn were compared to Monte Carlo results modelling decreasing cortical thickness to determine at what point we should expect to see ventricular contamination in the cerebral NIRS signal. Examining these data, we will begin to determine at what cortical thickness the assumption of light propagation through only brain is no longer valid within PHVD neonatal patients.

\section{METHODS}

\section{Patients}

Neonates with IVH were enrolled into the study following a positive diagnosis on a clinical head ultrasound. Study protocol has been approved by the local Research Ethics Board. To date, we have enrolled 54 patients and, of those, 10 required ventricle taps. Patients in the NICU with ventricle dilation for other reasons such as spina bifida, meningitis, or from a congenital cause were also enrolled in the study. The severity of the IVH was graded based on the scale by Papile et al.[11] which is the current clinical standard, with grade I-III describing increasing severity of hemorrhage contained in the ventricle, and a grade IV describing a hemorrhage into the periventricular brain region.

\section{Cortical Thickness through 3D Ultrasound imaging}

To determine the severity of the PHVD, 3D ultrasound images were acquired every time a NIRS cerebral measurement was performed. The 3D US system has previously been described and validated.[12] A coronal slice at the level of the Foramen of Monro was selected and cortical thickness (CTh) measurements were estimated (Fig. 1).

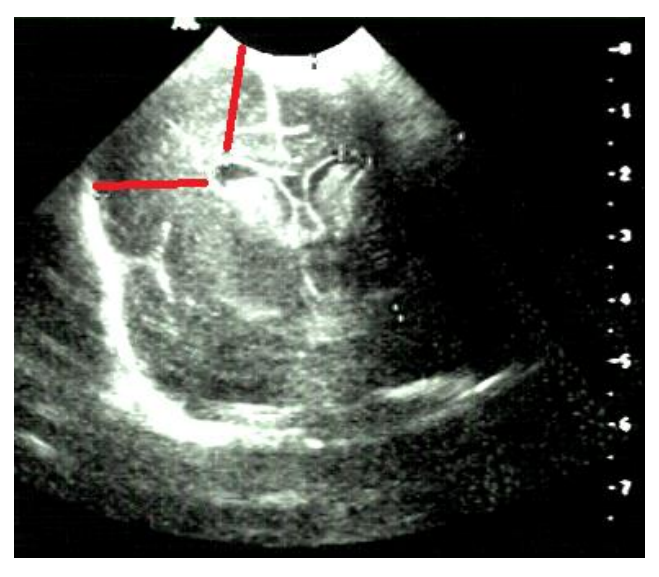

Figure 1 - Ultrasound image of a preterm neonatal brain in the coronal plane at the level of the Foramen of Monro showing a bilateral bleed in the lateral ventricles along with mild ventricle dilation. Estimates of cortical thickness indicated by lines on the image. 


\section{Cerebral spectral measurements from CW system}

A hyperspectral CW-NIRS system was used at the patients' bedside to obtain cerebral spectral measurements during the first month of life, usually during the second week of life. This system has previously been used in a neonatal piglet model[13, 14] and neonatal patients at our center.[15] The optical fibers were attached to the head either using a fiber holder headband, or using the CPAP cap to support the fiber holder. We obtained broadband spectra from 600-980 nm at approximately $0.3 \mathrm{~nm}$ spectral resolution using a holographic grating coupled to a cooled CCD. Tissue spectra were normalized to $960 \mathrm{~nm}$ (the 'water peak') as the concentration of water in the neonatal head is relatively stable around $90 \%[16]$ in comparison to the other chromophores.

\section{CSF spectral measurements from CW system}

If an IVH patient enrolled in the study required a VT for clinical reasons, the CSF removed during the procedure was analyzed prior to being discarded. The reference signal was acquired from a $1 \times 1 \times 3 \mathrm{~cm}$ polymethacrylate cuvette (SigmaAldrich Canada, Oakville, ON) prior to filling the cuvette with CSF, after which the absorption signal was recorded from the sample. Assuming negligible scattering in the CSF sample, the Beer-Lambert law was used to measure the absorption spectrum of each sample. To date we have collected 12 CSF samples.

\section{Monte Carlo Simulations}

Simulations of light propagation through the neonatal head were performed with Monte Carlo eXtreme[17] using a twolayer model of brain tissue and CSF to estimate the expected signal contamination from the latter for a range of cortical thicknesses. Simulations were performed with assumed tissue properties shown in Table 1 at an emission wavelength of $800 \mathrm{~nm}$ [18-20] and a source detector distance of $3 \mathrm{~cm}$. For the purpose of this study, the properties of the cortex were taken to be an average of published white and gray matter values, and ventricular CSF was assumed to be relatively clear, with low scattering and no bbp. These characteristics would represents CSF samples from either normal patients or patients in which the bbps from the IVH would have broken down. Mean photon pathlengths in both brain and ventricle layers were recorded after 20 simulations of $10^{8}$ photons each.

Table 1 - Optical properties for simulations. Cortical thickness varied between 5-25 mm

\begin{tabular}{|l|l|l|l|l|}
\hline Tissue & $\mu_{\mathrm{a}}\left(\mathrm{mm}^{-1}\right)$ & $\mu_{\mathrm{s}}{ }^{\prime}\left(\mathrm{mm}^{-1}\right)$ & $\mathrm{g}$ & $\mathrm{n}$ \\
\hline Cortex & 0.0425 & 0.8 & 0.9 & 1.3 \\
\hline CSF & 0.0041 & 0.032 & 0.9 & 1.3 \\
\hline
\end{tabular}

\section{RESULTS}

\section{Cortical Thickness Measurements}

During the neonatal period following clinical diagnosis of IVH, the thickness of the cortex ranged from 0.80 to $2.20 \mathrm{~cm}$ (Fig. 2). The average CTh measured by 3D US was $1.58 \pm 0.34 \mathrm{~cm}$. Generally, patients with more severe IVH (grade II and III) had lowered CTh. Grade IV IVH tended to have markedly lower CTh if the hemorrhagic region became cystic (such as in the patient with a CTh of $0.8 \mathrm{~cm}$ ), although cystic development was not always present. We used corrected gestational age (GA) as a measure of prematurity, with a GA of 37-40 weeks considered a 'full term' infant. Patients ranged between 25-35 weeks GA. Generally, patients born more preterm had lowered CTh, since they had smaller heads (Fig 2). 


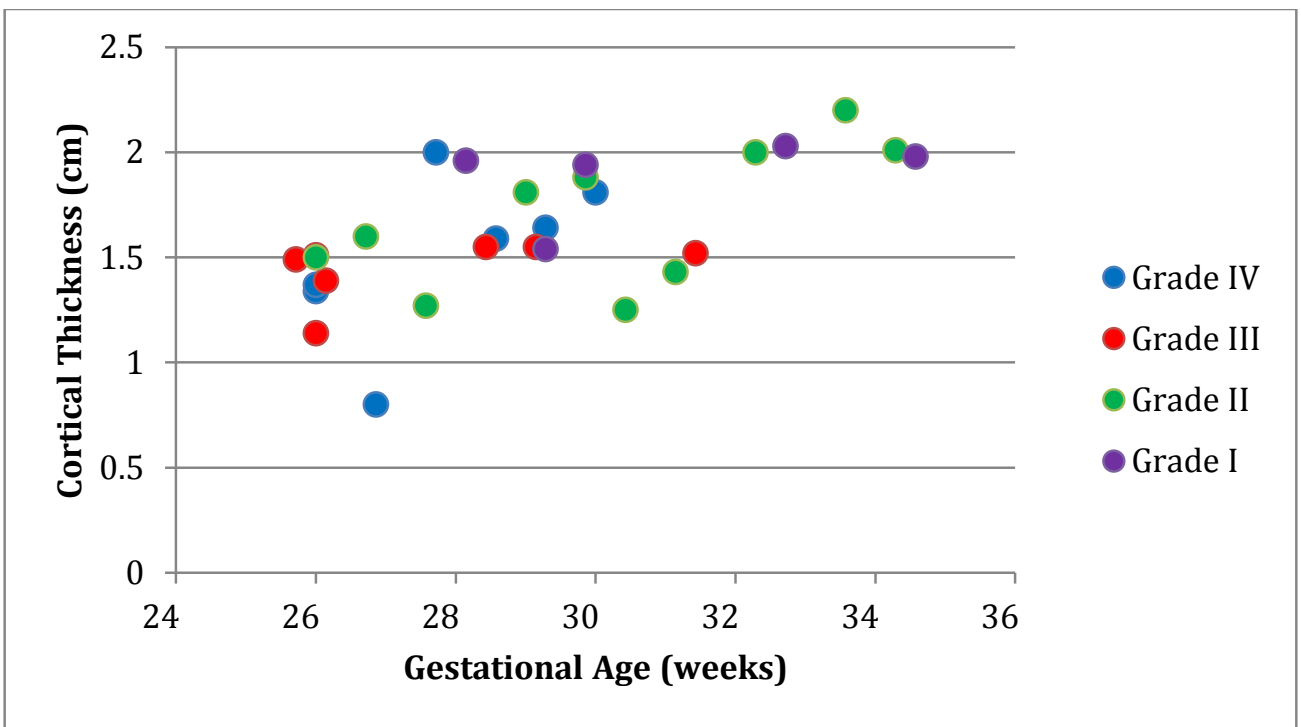

Figure 2 - Cortical thickness measurements of very low birth weight neonates with IVH obtained from coronal slices at the level of the Foramen of Monro from 3D US images taken < 30 minutes before NIRS acquisition.

\section{Cerebral Broadband NIRS Spectra - Patients with mild IVH}

Patient descriptions during baseline NIRS are shown in Table 2. Patients who had cortical thicknesses above $1.5 \mathrm{~cm}$ had similar looking spectra but once the CTh was under $1.3 \mathrm{~cm}$, a noticeable 'shift' in the spectra in the lower wavelengths was evident (Fig. 3). These patients usually had mild ventricle dilation as seen in Fig 4; however, most patients eventually had spontaneous resolution of this ventricle dilation.

Table 2 - Patient Characteristics for those diagnosed with mild IVH

\begin{tabular}{|l|l|l|l|l|}
\hline Patient & Age (days) & GA (weeks) & $\begin{array}{l}\text { IVH } \\
\text { Grade }\end{array}$ & CTh (cm) \\
\hline 1 & 6 & 26 & II & 1.50 \\
\hline 2 & 17 & $334 / 7$ & II & 2.20 \\
\hline 3 & 15 & $274 / 7$ & II & 1.27 \\
\hline 4 & 13 & $303 / 7$ & II & 1.25 \\
\hline
\end{tabular}




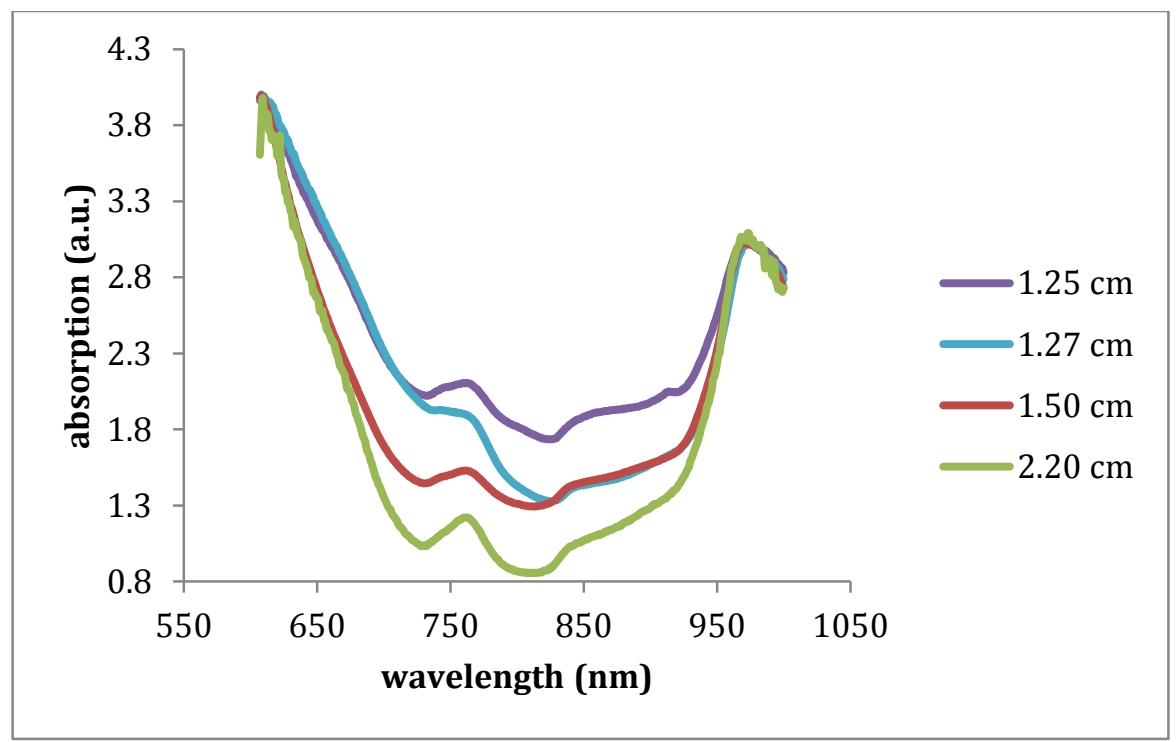

Figure 3 - Spectra from four neonates with grade II IVH who had differing levels of ventricle dilation. Cortical thickness indicated in the legend. All spectra were normalized to absorbance at $960 \mathrm{~nm}$.
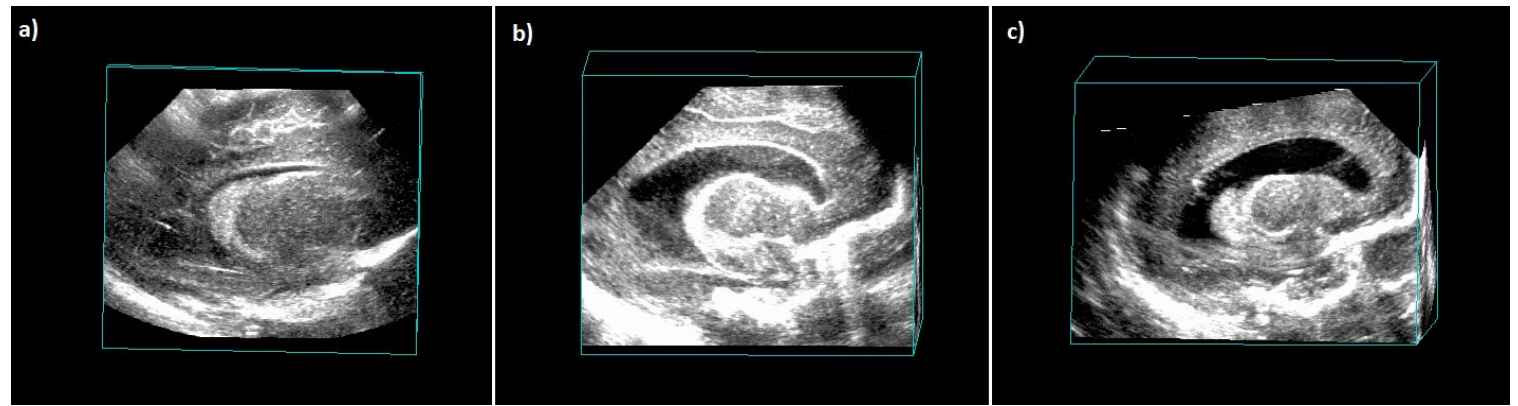

Figure 4 - 3D ultrasound images cut through the para-sagittal plane of patients diagnosed with mild IVH (grade II). Cortical Thickness was measured to be a) $2.20 \mathrm{~cm} \mathrm{~b}) 1.50 \mathrm{~cm}$, and c) $1.27 \mathrm{~cm}$.

\section{Cerebral Broadband NIRS Spectra - Patients with severe IVH and PHVD}

For PHVD with marked ventricle dilation, as seen in the ultrasound images, their spectra has more absorbance in lower wavelengths (Fig. 5). A representative case study of a neonate with bilateral grade III IVH is illustrated in Fig. 6 . The same patient's CSF spectra is seen in Figure 7, showing marked decreases in absorption over time as the bbp is cleared from the ventricles. This 'clearance' over time of the bbp can be seen visually in the photographs of the CSF samples in Figure 8, as the CSF returns slowly to a 'normal' clear CSF (not shown). 


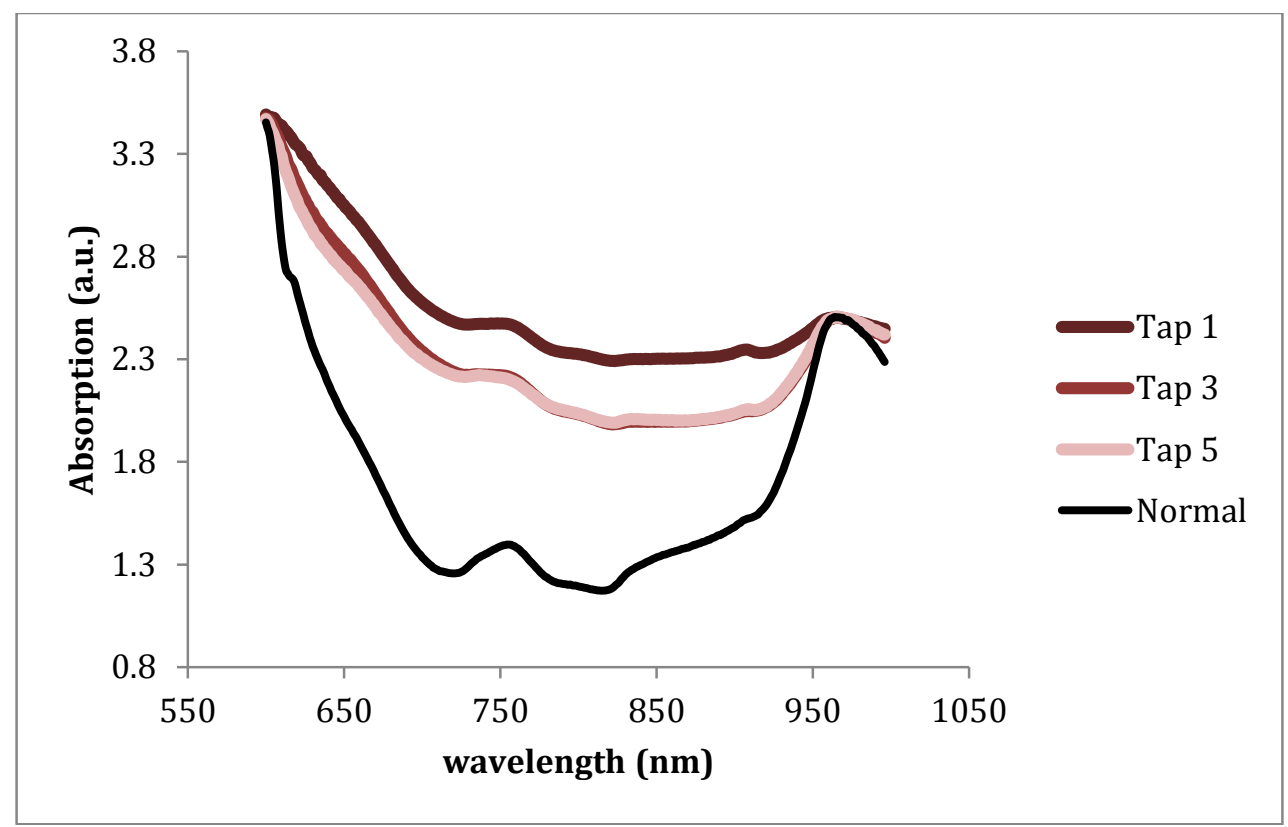

Figure 5 - b) Spectra from one infant, diagnosed with bilateral grade III IVH, who underwent three interventions. The 'normal' brain is mean spectrum from 5 patients without IVH. All spectra were normalized to absorbance at $960 \mathrm{~nm}$.
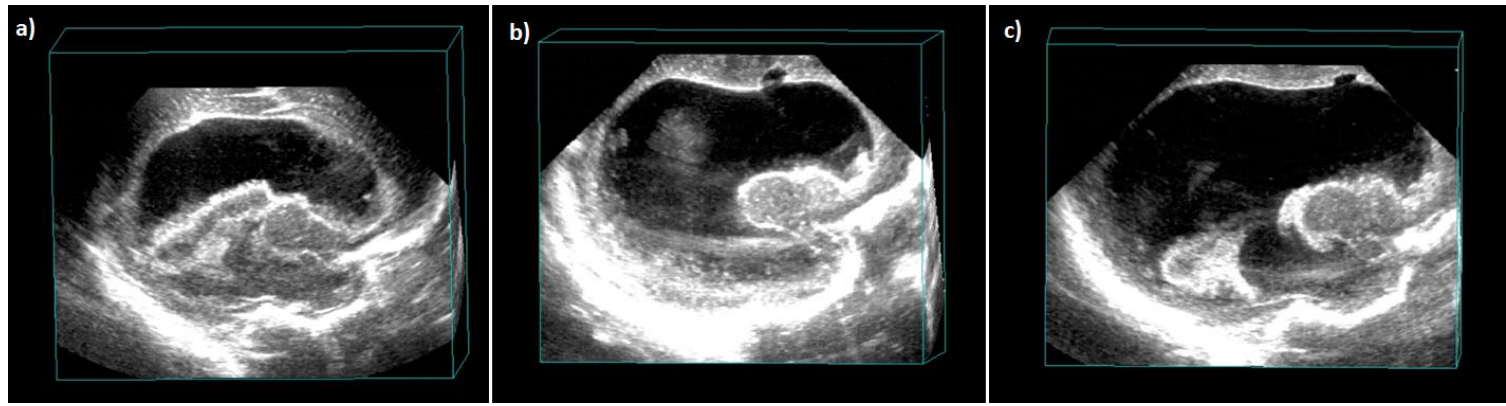

Figure 6 - 3D ultrasound images cut through the para-sagittal plane of from the example tapped neonate. Cortical thickness was measured to be a) $1.0 \mathrm{~cm}$ at tap 1 , b) $1.0-0.8 \mathrm{~cm}$ at tap 3 , and c) $0.8-0.6 \mathrm{~cm}$ at tap 5 . 


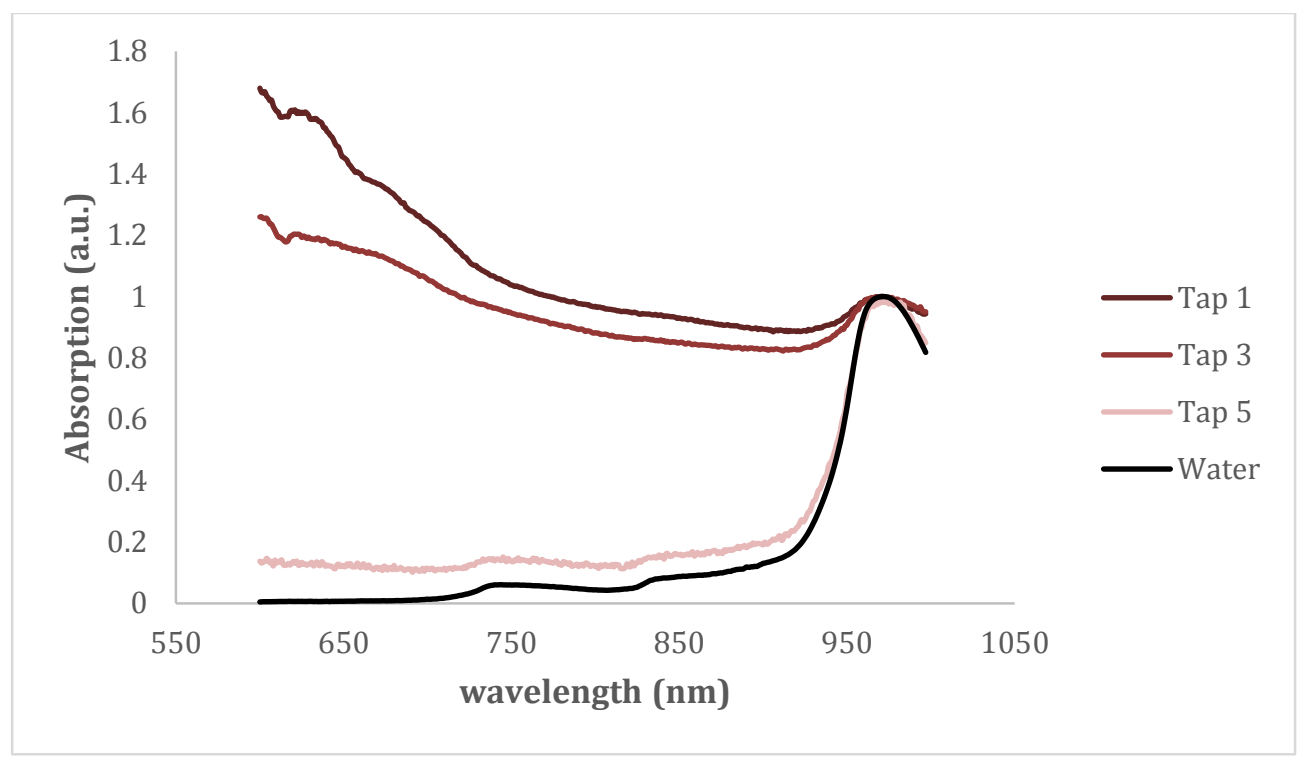

Figure 7 - CSF Spectra from the $1^{\text {st }}, 3^{\text {rd }}$ and $5^{\text {th }}$ ventricle tap of an IVH patient undergoing serial ventricle taps to relieve increased ICP. Spectra were normalized to $960 \mathrm{~nm}$.
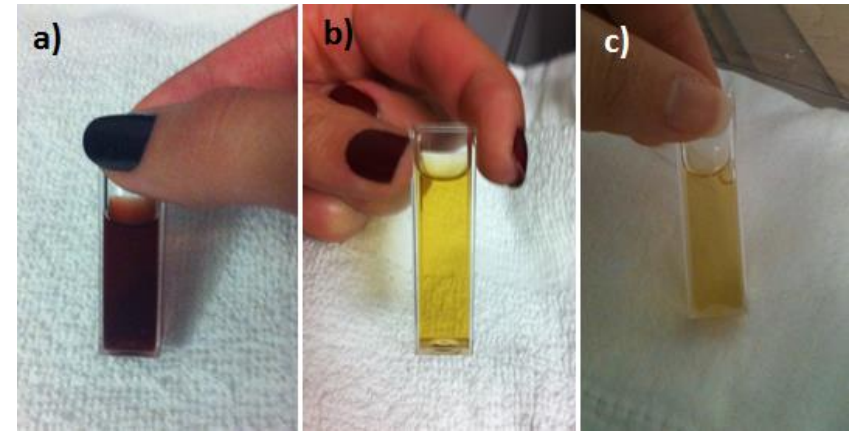

Figure 8 - Photographs of tapped CSF from a single neonate diagnosed with bilateral grade III IVH who underwent serial ventricle taps over the course of 3 weeks until a ventricular drain was implanted around 5 weeks of life. a) first tapped sample at 16 days of life b) third tap at 28 days of life and c) fifth tap at 36 days of life.

\section{Monte Carlo Simulations}

Monte Carlo simulations showed that for clear CSF, there was negligible difference between total pathlength and pathlength travelled through cortex for large Cth $(>2 \mathrm{~cm}$ ) (Fig. 9). However, at cortical thickness less than $1.3 \mathrm{~cm}$ the contribution of the mean pathlength was almost $10 \%$ from CSF. At the smallest observed cortical thickness in this present study $(0.8 \mathrm{~cm})$ the CSF contributed to $43 \%$ of the DP. 


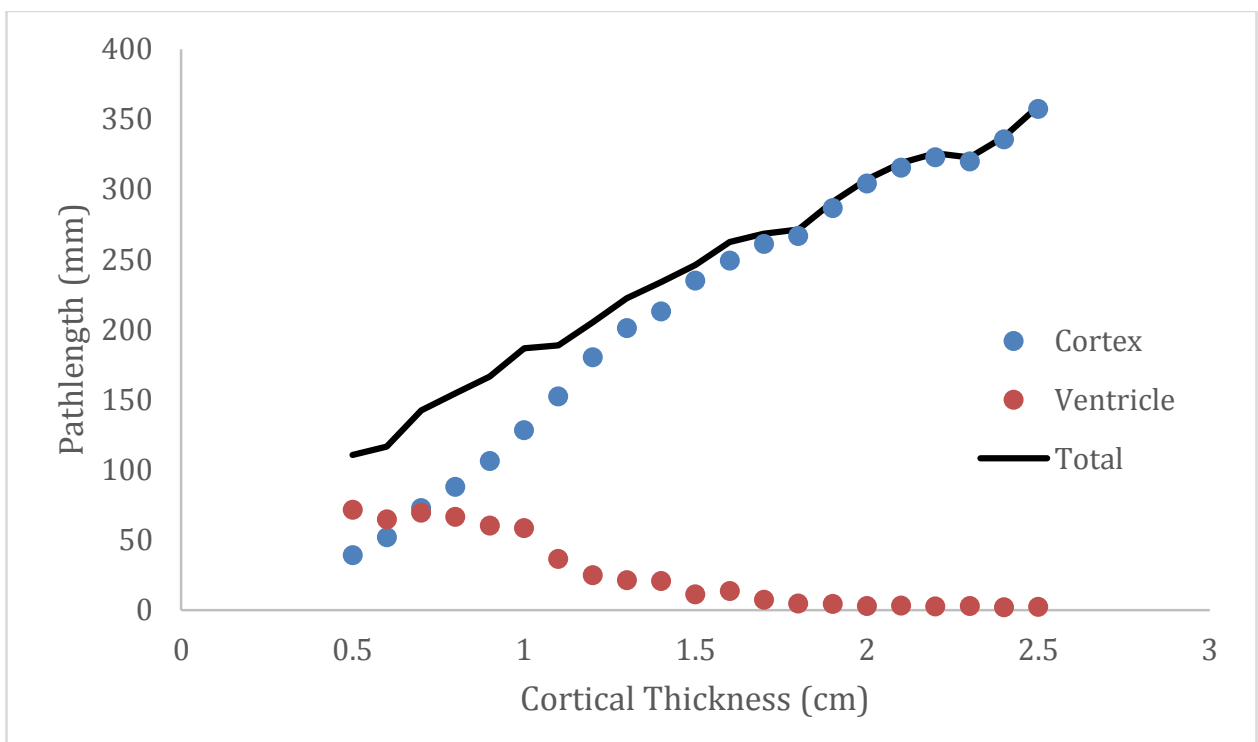

Figure 9 - Differential pathlengths for simulations over a range of cortical thicknesses. Mean total pathlength is indicated by the black line, while the partial pathlengths for brain and ventricular space are shown by the colored symbols. Simulations were performed for a wavelength of $800 \mathrm{~nm}$ and a source-detector separation of $3 \mathrm{~cm}$.

\section{DISCUSSION}

We have shown qualitatively that there appears to be noticeable ventricular contamination in the absorption signal from IVH patients with enlarged ventricles (Figs. 3, 5); however, this effect was not as noticeable in patients with thickness greater than $1.5 \mathrm{~cm}$. This was corroborated by the results of the Monte Carlo simulations that showed a steep increase in signal contribution from the ventricles once the cortical thickness fell below $1.3 \mathrm{~cm}$ (Fig. 9). Since many clinical systems advice against the use in all IVH patients, perhaps these results show that there is a sub-section of IVH patients who have mild or no ventricle dilation who would be candidates for NIRS monitoring in spite of the IVH.

That said, it should be noted that IVH patients with significant ventricle dilation, so called 'post hemorrhagic hydrocephalus' are the patients at greatest risk of neurological impairment later in life. These are the patients who would likely benefit the most from neuromonitoring during the neonatal period in order to better manage their condition through therapies and surgeries aimed at alleviating elevated ICP. As such, the results from this study provide some insight into how a two-layer model of the brain/ventricles could assist in analyzing NIRS signal from PHH patients in order to more accurately monitor brain oxygenation despite variations in ventricle volume.

A limitation in the current study was the simulations were only performed using optical properties approximating clear CSF with no bbp. Consequently most of the light reaching the ventricle space would not be scattered back into the tissue. Likely, a model for darker, more absorbing CSF, with greater scattering properties would show different effects. Likely quantification of scattering in CSF with significant bbp is also required, which is currently under investigation. Another limitation was the cortical thickness measurements were not made at exactly the area interrogated by the NIRS probes because of the limited field of view obtained through the fontanels used to obtain the US images. The specific location for US imaging was chosen to capture the ventricle volume; however, other locations could be selected to image the cortical region interrogated by NIRS.

In conclusion, considering there have been many studies exploring the ability to improve the sensitivity to the brain by the use of 'image guided' approaches, such as incorporating a priori information in the analysis of NIRS measurements, even a simple measure of cortical thickness from US should greatly improve the accuracy of NIRS when applied to neonates with PHH. 


\section{ACKNOWLEDGEMENTS}

This study was facilitated with the support of Neonatal-Perinatal Medicine at Victoria Hospital, as well as all the NICU physicians and nurses. Many thanks are also required for the ongoing support of the ultrasound technicians involved in the study as well as the Department of Radiology at Victoria hospital. We would also like to extend a special thanks to all of the families who graciously consented to be included in this research, for without them, none of this would have been possible. The authors would like to acknowledge the funding support from Canadian Institutes of Health Research (CIHR), the Canada Research Chairs (CRC) Program, Lawson Health Research Institute, and the Academic Medical Organization of Southwestern Ontario (AMOSO). This work was also supported by a personnel award to K St. Lawrence from the Heart and Stroke Foundation, Ontario Provincial Office

\section{REFERENCES}

[1] A. R. Synnes, L. Y. Chien, A. Peliowski et al., "Variations in intraventricular hemorrhage incidence rates among Canadian neonatal intensive care units," J Pediatr, 138(4), 525-31 (2001).

[2] J. M. Perlman, J. B. McMenamin, and J. J. Volpe, "Fluctuating cerebral blood-flow velocity in respiratorydistress syndrome. Relation to the development of intraventricular hemorrhage," N Engl J Med, 309(4), 204-9 (1983).

[3] E. Calisici, Z. Eras, M. Y. Oncel et al., "Neurodevelopmental outcomes of premature infants with severe intraventricular hemorrhage," J Matern Fetal Neonatal Med, 1-6 (2014).

[4] S. Bolisetty, A. Dhawan, M. Abdel-Latif et al., "Intraventricular hemorrhage and neurodevelopmental outcomes in extreme preterm infants," Pediatrics, 133(1), 55-62 (2014).

[5] M. J. Brouwer, L. S. de Vries, F. Groenendaal et al., "New reference values for the neonatal cerebral ventricles," Radiology, 262(1), 224-33 (2012).

[6] A. Whitelaw, and K. Aquilina, "Management of posthaemorrhagic ventricular dilatation," Arch Dis Child Fetal Neonatal Ed, 97(3), F229-3 (2012).

[7] T. Alderliesten, P. M. Lemmers, J. J. Smarius et al., "Cerebral oxygenation, extraction, and autoregulation in very preterm infants who develop peri-intraventricular hemorrhage," J Pediatr, 162(4), 698-704.e2 (2013).

[8] S. Noori, M. McCoy, M. P. Anderson et al., "Changes in cardiac function and cerebral blood flow in relation to peri/intraventricular hemorrhage in extremely preterm infants," J Pediatr, 164(2), 264-70.e1-3 (2014).

[9] E. A. Verhagen, H. J. Ter Horst, P. Keating et al., "Cerebral oxygenation in preterm infants with germinal matrix-intraventricular hemorrhages," Stroke, 41(12), 2901-7 (2010).

[10] J. S. Soul, E. Eichenwald, G. Walter et al., "CSF removal in infantile posthemorrhagic hydrocephalus results in significant improvement in cerebral hemodynamics," Pediatr Res, 55(5), 872-6 (2004).

[11] L. A. Papile, J. Burstein, R. Burstein et al., "Incidence and evolution of subependymal and intraventricular hemorrhage: a study of infants with birth weights less than 1,500 gm," J Pediatr, 92(4), 529-34 (1978).

[12] J. Kishimoto, S. de Ribaupierre, D. S. Lee et al., "3D ultrasound system to investigate intraventricular hemorrhage in preterm neonates," Phys Med Biol, 58(21), 7513-26 (2013).

[13] D. W. Brown, J. Hadway, and T. Y. Lee, "Near-infrared spectroscopy measurement of oxygen extraction fraction and cerebral metabolic rate of oxygen in newborn piglets," Pediatr Res, 54(6), 861-7 (2003).

[14] K. M. Tichauer, D. W. Brown, J. Hadway et al., "Near-infrared spectroscopy measurements of cerebral blood flow and oxygen consumption following hypoxia-ischemia in newborn piglets," J Appl Physiol (1985), 100(3), 850-7 (2006).

[15] R. Arora, M. Ridha, D. S. Lee et al., "Preservation of the metabolic rate of oxygen in preterm infants during indomethacin therapy for closure of the ductus arteriosus," Pediatr Res, 73(6), 713-8 (2013).

[16] J. Dobbing, and J. Sands, "Quantitative growth and development of human brain," Arch Dis Child, 48(10), 75767 (1973).

[17] Q. Fang, and D. A. Boas, "Monte Carlo simulation of photon migration in 3D turbid media accelerated by graphics processing units," Opt Express, 17(22), 20178-90 (2009).

[18] A. Custo, W. M. Wells, 3rd, A. H. Barnett et al., "Effective scattering coefficient of the cerebral spinal fluid in adult head models for diffuse optical imaging," Appl Opt, 45(19), 4747-55 (2006).

[19] M. Dehaes, K. Kazemi, M. Pelegrini-Issac et al., "Quantitative effect of the neonatal fontanel on synthetic near infrared spectroscopy measurements," Hum Brain Mapp, 34(4), 878-89 (2013).

[20] Y. Fukui, Y. Ajichi, and E. Okada, "Monte Carlo prediction of near-infrared light propagation in realistic adult and neonatal head models," Appl Opt, 42(16), 2881-7 (2003). 